\title{
First adult case of sporadic localized glomerulocystic kidney mimicking a tumor
}

\author{
FUMIYOSHI KOJIMA $^{1}$, MITSUAKI ISHIDA ${ }^{2}$, YUICHI TSUJIMOTO ${ }^{3}$, MASAHIRO HOSOMI ${ }^{3}$, \\ KINOUCHI TOSHIAKI ${ }^{3}$ and HIDETOSHI OKABE ${ }^{2}$ \\ ${ }^{1}$ Department of Diagnostic Pathology, Saiseikai Senri Hospital, Suita, Osaka 565-0862; \\ ${ }^{2}$ Department of Clinical Laboratory Medicine and Division of Diagnostic Pathology, \\ Shiga University of Medical Science, Otsu, Shiga 520-2192; ${ }^{3}$ Department of Urology, \\ Saiseikai Senri Hospital, Suita, Osaka 565-0862, Japan
}

Received March 15, 2014; Accepted December 3, 2014

DOI: $10.3892 / \mathrm{ol} .2015 .3060$

\begin{abstract}
Glomerulocystic kidneys (GCKs) are mainly observed in infants and young children, and are characterized by the cystic dilatation of Bowman's space to form glomerular cysts (GCs). GCKs are associated with various conditions. Additionally, the cystogenesis of GCKs remains controversial. The present study describes a rare adult case of a sporadic localized GCK that radiologically mimicked a multilocular cystic tumor, and analyses the features of GC. A 42-year-old male with hematuria underwent a right partial nephrectomy for a cystic mass. The majority of the cyst was distributed in the cortex and contained a single collapsed glomerulus. Using serial sections, narrow and serpiginous proximal tubules that continued to the GCs were detected. These findings suggested that obliteration at the glomerulotubular junction was not the primary cause of GC in this case. To the best of our knowledge, this is the first adult case of a sporadic localized GCK mimicking a tumor. Unnecessary surgical procedures may be avoided by careful evaluation of computed tomography scans and magnetic resonance imaging, although localized GCKs are quite rare.
\end{abstract}

\section{Introduction}

Glomerulocystic kidneys (GCKs) are characterized by the cystic dilatation of Bowman's space to form glomerular cysts (GCs), and are mainly observed in infants and young children in association with the following conditions: Hereditary polycystic kidney disease, tuberous sclerosis, renal dysplasia and renal ischemia, and certain medications,

Correspondence to: Dr Fumiyoshi Kojima, Department of Diagnostic Pathology, Saiseikai Senri Hospital, 1-1-6 Tsukumodai, Suita, Osaka 565-0862, Japan

E-mail: fumiyosh@belle.shiga-med.ac.jp

Key words: glomerular cyst, glomerulocystic kidney, adult, localized including lithium (1). To the best of our knowledge, only 35 cases of GCKs have been reported in adults worldwide (1-3). The majority of these patients presented with decreased renal function and subsequently received hemodialysis treatment, however they eventually progressed to end-stage renal disease $(2,3)$. However, a few cases had normal renal function or were asymptomatic (1-4). GCKs are generally diagnosed by open renal biopsy. The lesion is not generally recognized as a neoplatic mass and cases of GCKs mimicking multilocular renal carcinoma are rare. The present study describes the first adult case of a sporadic localized GCK that presented as a cystic mass mimicking a neoplasm, and provides an analysis of the features of GC. Informed consent was obtained from the patient's family.

\section{Case report}

An asymptomatic 42-year-old male presented to Saiseikai Senri Hospital (Osaka, Japan) was revealed to have microscopic hematuria following a medical check-up. The patient was revealed to have a localized nest of multilocular cysts, without any expansile nodules, which measured $2 \times 4 \mathrm{~cm}$ in diameter. The cystic mass was detected in the lower pole of the right kidney by abdominal ultrasonography, contrast-enhanced computed tomography (CT) (Fig. 1A) and gadolinium-enhanced magnetic resonance imaging (MRI) (Fig. 1B). Contrast-enhanced CT revealed enhancement in the lesion [Bosniak classification category III (4)]. Cystic renal cell carcinoma could not be ruled out, therefore, a right partial nephrectomy was performed.

Grossly, the lesion was composed of multiple cysts filled with serous fluid, each measuring $\leq 8 \mathrm{~mm}$ in maximum diameter, and was distributed in the cortex of the resected kidney (Fig. 2). A small calculus measuring $2 \mathrm{~mm}$ in diameter was deposited in the outer medulla directly under the lesion. Microscopically, the majority of the cysts were lined by a single layer of flattened epithelium and a collapsed glomerulus was evident (Fig. 2C). In the corticomedullary junction, a few cysts lined by epithelial membrane antigenand CK34 3 E12-positive cuboidal epithelium were suggestive of a derivation from the distal tubule or collecting duct. This 
A

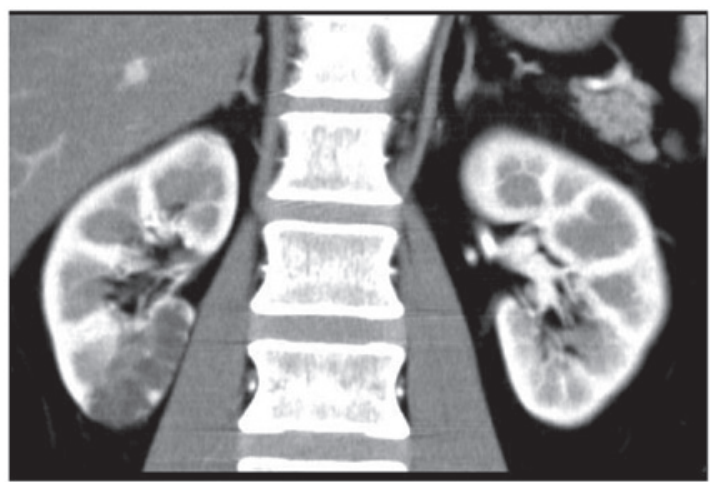

B

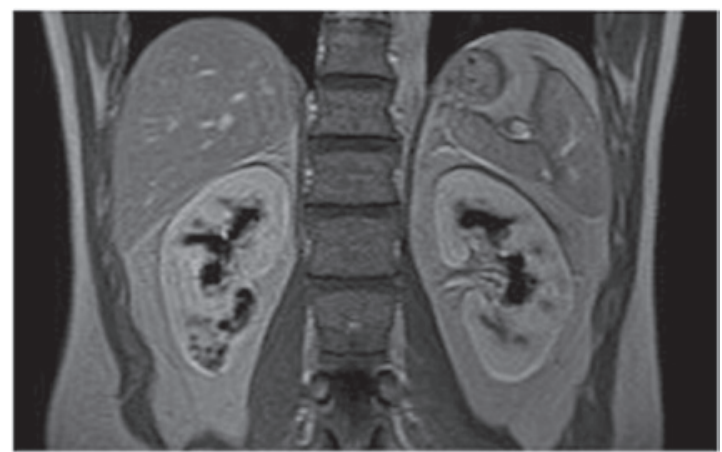

Figure 1. (A) Contrast-enhanced computed tomography and (B) magnetic resonance imaging showing a $2 \times 4-\mathrm{cm}$ multilocular lesion with enhancement in the right lower pole of kidney.

lesion was diagnosed as a sporadic case, as no family history or clinical history was found that was associated with GCKs.

A serial section study was performed for light microscopy to examine the GTJ. A total of $2504-\mu \mathrm{m}$ thick sections and $1002-\mu \mathrm{m}$ thick sections were stained with hematoxylin and eosin (HE) and Periodic acid-Schiff (PAS), respectively. A GC was defined as Bowman's capsule dilation of more than twice the diameter of a normal Bowman's capsule $(320 \mu \mathrm{m})$, as described in a previous study (1). Each single specimen contained 50-80 cysts. Eight and six GTJs from 30 examined GCs were detected in these HE- and PAS-stained sections, respectively. These sections revealed a connection between the GCs and the proximal tubule, although the connected tubule became narrow and serpiginous (Fig. 3). The patient was lost to follow-up.

\section{Discussion}

Generally, GCKs diffusely involve the bilateral kidneys in infants and young children. To the best of our knowledge, this is the first adult case of a sporadic GCK mimicking a tumor. Retrospectively, the enhancement of this lesion that was identified on $\mathrm{CT}$ was determined to be that of a residual normal cortex. Radiologically, the lesion was almost entirely localized in the cortex, without renal surface deformity or protrusion from the kidney, and did not have the capsular and peritumoral change associated with invasive or expansive growth. These findings suggest that it was a non-neoplastic lesion. Unnecessary surgery may be avoided in future cases by careful evaluation of the CT and MRI, although localized GCKs are quite rare.
A

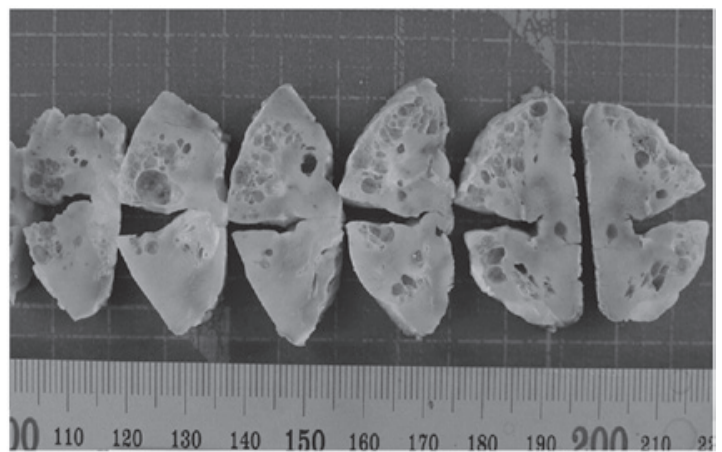

B

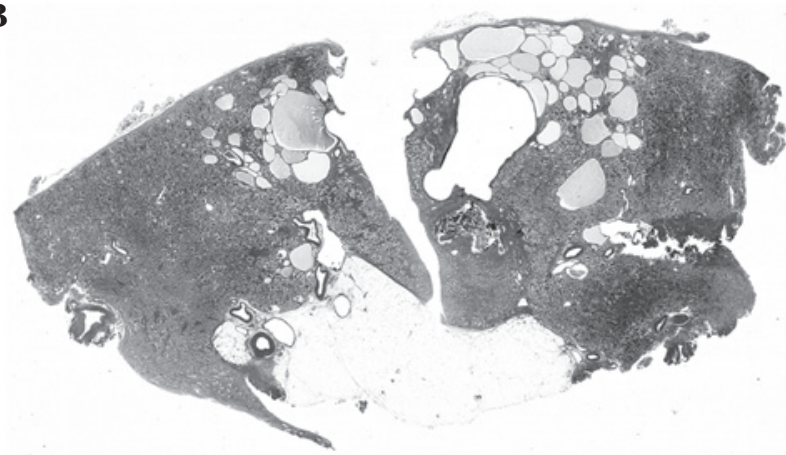

C

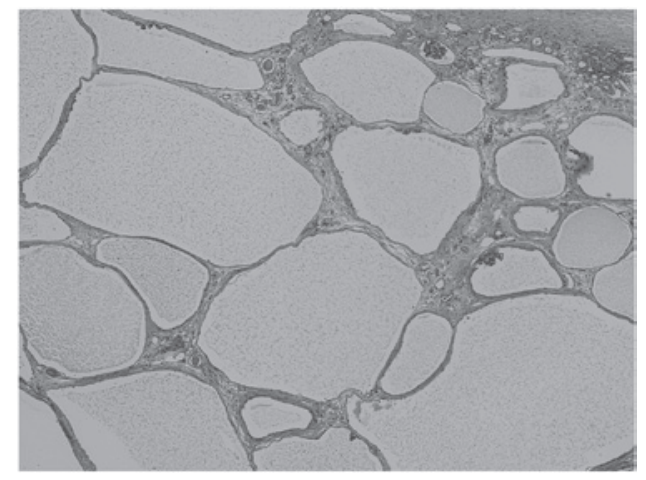

Figure 2. Multilocular cystic lesion on the (A) cut surface and (B) whole mount section of the resected kidney. The cysts varied in size, measuring $8 \mathrm{~mm}$ in maximum diameter, and were distributed in the cortex. (C) Low-power view showing glomerular cysts and a collapsed glomerulus lined by flattened epithelium (HE staining; magnification, $\mathrm{x} 40$ ).

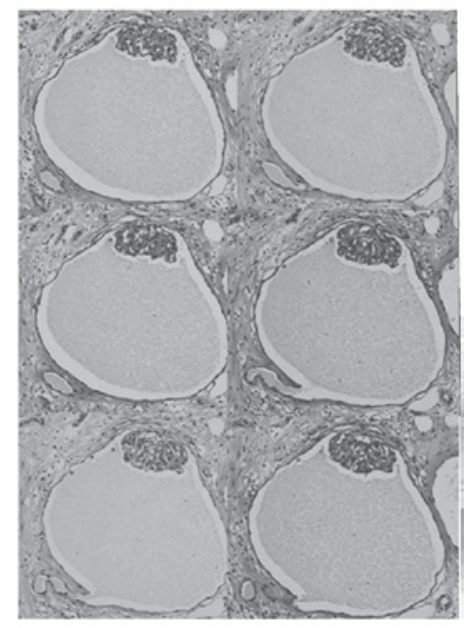

Figure 3. Serial section of glomerular cysts. A single collapsed glomerulus and the glomerulotubular junction. The proximal tubule connecting to the GC was narrow and serpiginous and the proximal tubular epithelium was flattened. Hematoxylin and eosin staining; magnification, x200 
Obliteration at the glomerulotubular junction (GTJ) has been assumed to be the cause of GCKs associated with several diseases, however, the exact cause remains controversial $(3,5,6)$. Hotta et al (5) used serial sections to demonstrate GTJ stenosis and suggested that periglomerular fibrosis induced stenosis of GTJ. By contrast, Liu et al (3) identified no stenosis or obstruction of the GTJ using serial sections. In the present case, a connection between the GCs and the proximal tubule was identified. These findings suggested that obliteration at the GTJ was not the primary cause of the GCs in the present case. Since this case was composed of large GCs with non-detectable surrounding proximal tubules, it was reasonable to assume that the GCs were composed of Bowman's capsule and part of the proximal tubule (7). We hypothesize that proliferation of the parietal cells and renal tubular cells, fluid accumulation and remodeling of the nephron (6) were the main causes of cyst development in the present case. As various conditions are associated with the formation of GC, such as hereditary polycystic kidney disease, tuberous sclerosis and renal dysplasia, we hypothesize that different pathogenic mechanisms of GC formation exist.

In summary, the present study reported an adult case of a sporadic localized GCK that resembled a cystic renal neoplasm, and provided analysis of its characteristic histopathological features using serial sections.

\section{Acknowledgements}

The authors would like to thank Mr. Masaru Nishino, Mrs. Noriko Yokozeki and Mr. Manabu Kobayashi (Department of Central Clinical Laboratory, Saiseikai Senri Hospital, Suita, Osaka, Japan) for their technical assistance in the serial sectioning.

\section{References}

1. Lennerz JK, Spence DC, Iskandar SS, Dehner LP and Liapis H: Glomerulocystic kidney: one hundred-year perspective. Arch Pathol Lab Med 134: 583-605, 2010.

2. Obata Y,Furuse A, Miyazaki M, Nishino T, Kawazu T, Kanamoto Y, Nishikido M, Taguchi T and Kohno S: Glomerulocystic kidney disease in an adult with enlarged kidneys: a case report and review of the literature. Clin Nephrol 75: 158-164, 2011.

3. Liu JS, Ishikawa I, Saito Y, Nakazawa T, Tomosugi N and Ishikawa Y: Digital glomerular reconstruction in a patient with a sporadic adult form of glomerulocystic kidney disease. Am J Kidney Dis 35: 216-220, 2000.

4. Bosniak MA: The Bosniak renal cyst classification: 25 years later. Radiology 262: 781-785, 2012.

5. Hotta O, Sato M, Furuta T and Taguma Y: Pathogenic role of glomerulo-tubular junction stenosis in glomerulocystic disease. Clin Nephrol 51: 177-180, 1999.

6. Liu JS, Ishikawa I, Saito Y, Nakazawa T, Tomosugi N and Ishikawa Y: Digital glomerular reconstruction in a patient with a sporadic adult form of glomerulocystic kidney disease. Am J Kidney Dis 35: 216-220, 2000.

7. Kanouzawa K, Tamura H, Matsumura O, Nagasawa R, Mitarai T, Isoda $\mathrm{K}$ and Yamanaka N: An adult case of glomerulocystic kidney disease. Nihon Jinzo Gakkai Shi 36: 762-768, 1994 (In Japanese). 